\title{
ENERGY FLOW: WAVE MOTION AND GEOMETRICAL OPTICS ${ }^{1}$
}

\author{
BY CATHLEEN S. MORAWETZ
}

\begin{abstract}
ABSTRACr. Energy distribution for solutions of the wave equation in the presence of a reflecting body can be investigated with varying degrees of refinement by using quadratic inequalities, Huyghens principle and geometrical optics. The relations between these properties and their validity in general cases is discussed and some of the simpler proofs outlined.
\end{abstract}

1. Introduction. This paper deals with the motion of conservative systems, i.e. systems whose total energy remains the same for all time. Energy in general is a positive functional of the instantaneous state of the system; for systems governed by linear equations energy is a positive definite quadratic form. Conservation of energy implies that if the initial state is zero, so are all subsequent and previous states; for linear systems we conclude that solutions are uniquely determined by their initial data. For solutions with nonzero initial states the conservation of energy furnishes an a priori bound which, when combined with orthogonal projection techniques, yields a proof of the existence of solutions with arbitrarily prescribed initial data with finite energy.

Thus for conservative systems we can prove rather easily the existence and uniqueness of solutions. Current research is directed at establishing more detailed properties of the way solutions evolve in time, in particular for systems located in an unbounded domain interest focuses on the following problems:

I. Energy flow. In the course of time where will the energy or amplitude of a solution be concentrated? Will it eventually diffuse to infinity?

II. Transport of singularities. If the data contain singularities how and where are they propagated?

III. Asymptotic description. What is the behavior of highly oscillatory solutions? Can they be described by a simplified theory? An

An address delivered at the 664th meeting of the American Mathematical Society, New York, N. Y., April 4, 1969, by invitation of the Committee to Select Hour Speakers for Eastern Sectional Meetings; received by the editors February 12, 1970.

AMS Subject Classifications. Primary *7850, *7805, 3509; Secondary 3576, 3516.

Key Words and Phrases. Energy flow, wave equation, reflecting body, transport of singularities, geometrical optics, scattering.

1 This paper represents results obtained at the Courant Institute of Mathematical Sciences, New York University, with the U. S. Army Research Office, Contract DA-31-124-ARO-D-365. 
example would be the geometrical optic description of wave motion.

IV. Scattering. What is the relation between the solutions for large negative times and large positive times? How does the behavior at infinity alter as the equation or domain is varied away from infinity?

For all the problems of physics with questions fitting into this simple list, the answers are basically known but lack mathematical proof except in special cases. The best investigated are those connected with Schrödinger's equation, Maxwell's equations and the classical wave equation. But unsolved problems abound in the theory of water waves. For example, if you rock a boat and then stop, clearly the energy is transported to infinity in waves but this answer is not mathematically established.

The theory for the classical wave equation also has had many gaps, some of which have been filled within the past few years. It is this theory that constitutes this paper. Apologies are due for its even narrower scope which concentrates on the work the author has been involved in, and cavalierly disregards the rest, in particular the intricate and difficult details of the diffraction work done by J. B. Keller and co-workers which revived the interest in this subject.

Thus we are henceforth dealing with solutions $u$ of

$$
\square u=u_{t t}-\Delta u=0
$$

in some infinite domain $\varepsilon$ to be specified later.

We define the energy at time $t$ in a subdomain $D$ of $\varepsilon$ to be

$$
E(t, D)=\int_{D}\left(|\nabla u|^{2}+u_{t}^{2}\right)|d x| .
$$

The Cauchy data are

$$
u=\phi, \quad u_{t}=\psi, \quad \text { for } t=0, \quad x \in \varepsilon .
$$

To complete the definition of the problem we would need to specify $\mathcal{E}$ and some appropriate boundary data on $\partial \mathcal{E}$, the boundary of $\mathcal{E}$, which will ensure that the total energy is conserved, i.e. $E(\mathcal{E}, t)$ $=$ constant.

2. Free space solutions. We first recollect the answers to our questions for the simplest problem where $\varepsilon$ is all of space. Suppose the Cauchy data have compact support $k$,

$$
\phi \equiv \psi \equiv 0 \quad \text { for }|x|>k .
$$

I. By Huyghens' principle, if the number of space dimensions is odd, 
or

$$
u \equiv 0 \quad \text { for }|x|<t+k
$$

$$
E(t, D) \equiv 0 \quad \text { for } t \text { large enough. }
$$

If $n$ is even

$$
u=O\left(t^{-1}\right), \quad E(t, D)=O\left(t^{-2}\right),
$$

for $t \rightarrow \infty$.

In both cases the main energy travels in a ring off to infinity since by a domain of dependence argument $u \equiv 0$ for $|x|>t+k$.

II. A singularity at a single point $x_{0}$ in the initial data propagates, for any dimension, along the characteristic cone $\left|x-x_{0}\right|= \pm t$ (with its integrated magnitude undiminished). A general singularity can be studied using either a fundamental solution or more simply the Fourier transform in space. The main result is a generalized Huyghens' principle, ${ }^{2}$ that is independent of dimension.

Let the support of the singular part of the Cauchy data be $k^{\prime}$ and the rest be $C^{\infty}$. Then $u \in C^{\infty}$ for $|x| \leqq t-k^{\prime}$ and $|x| \geqq t+k^{\prime}$.

III. The asymptotic question is very easily answered. The periodic solutions $U e^{i \lambda t}$ satisfy the reduced equation $\Delta U+\lambda^{2} U=0$ and their complete behavior is given by using the fundamental solutions or the plane waves for integral representations and evaluating the integrals asymptotically for large $\lambda$.

IV. The behavior of solutions of $\square u=0$ at large distance and for large times is given by

$$
\begin{aligned}
u & \sim r^{-n / 2} f(\Omega, r-t), & & t \rightarrow \infty \\
& \sim r^{-n / 2} g(\Omega, r+t), & & t \rightarrow \infty
\end{aligned}
$$

where $\Omega$ represents the angular variables.

The differential equation has the effect of a mapping $\mathfrak{T}, g=\mathfrak{T} f$.

The answer to the last part of the scattering question depends on what one does to the equation. It is easy to construct weak perturbations of the equation ${ }^{3}$ so that this behavior is preserved but the mapping, say $\mathfrak{T}^{\prime}$, is different. Then the perturbed equation and its properties can be completely analyzed by studying the scattering operator $\mathfrak{T}^{-1} \mathfrak{M}^{\prime}$. The perturbation we are concerned with in the future consists in cutting a finite region out of the infinite domain.

3. The boundary value problem. Let $\varepsilon$ be the domain outside a finite body $B=\partial \mathcal{E}$ on which we prescribe an energy-conserving boundary condition

${ }^{2}$ See Courant and Hilbert[1, pp. 735-736].

${ }^{3}$ A class of nonlinear perturbations can also be treated, see Strauss [2]. 


$$
u=0 \text { or } \partial u / \partial n=0 .
$$

We can also consider the commonly occurring condition $\partial u / \partial n+\gamma n$ $=0, \gamma(x) \geqq 0$, which conserves $E^{\prime}=E(\varepsilon, t)+\int_{\Theta} \gamma u^{2} d \sigma$. Known results are similar to the Neumann problem.

In all cases the proof of energy conservation is given for odd dimensions by differentiating the energy, using the differential equation, integrating by parts and applying the boundary condition.

I. The most general result on energy flow is that $E(t, D) \rightarrow 0$ as $t \rightarrow \infty$. This was first proved for odd dimensions by Lax and Phillips [3]. It was also proved by establishing the existence of a wave operator for all dimensions by Schenk [4] and by the limiting absorption principle by Eidus [5]. The solutions are defined only in the distribution sense but the energy, given by the initial data

$$
E(t, \varepsilon)=\int\left(|\nabla \phi|^{2}+\psi^{2}\right)|d x|
$$

must be bounded.

There also exist indented bodies for which this is the best result, see Ralston [6], in the sense that by choosing the initial data sufficiently roughly one can have $E(t, D) / E(t, \varepsilon)$ arbitrarily close to 1 for arbitrarily large times.

On the other hand, if the body is star-shaped or close to it and the boundary condition is

$$
u=0 \quad \text { on } B \quad \text { (the reflection condition) }
$$

the decay of energy is rapid. Reflecting the power of Huyghens' principle in odd dimensions one has

$$
E(t, D)=O\left(e^{-\alpha t}\right) \quad \text { for } n \text { odd, }
$$

while

$$
E(t, D)=O\left(t^{-2}\right) \quad \text { for } n \text { even. }
$$

One sees this result easily for spheres by separating variables, see Wilcox [7]. For the general three-dimensional case using semigroups see Lax, Morawetz and Phillips [8] and without semigroups Morawetz [24]; the even dimensional result is contained in [9].

II. In connection with the transport of singularities we may suppose the singularity is isolated initially at $x_{0}$. One expects from special cases, see [10], that, after propagating to the body along the characteristic cone it is reflected along the envelope of the reflected characteristic cones. Equivalently, the singularity distributes itself and runs along the rays from the source $x_{0}$ to the body. There 
it is completely reflected along the customary reflected ray and behind the body, in its shadow, there are no singularities. This generalized Huyghens' principle has been proved for reflecting convex three-dimensional bodies provided the initial singularity is away from the body [11].

This result can be used via the Riemann function, see [12] and [13], to prove the following theorem which is also a weak generalized Huyghens' principle:

Smoothness Theorem. If $B$ is convex and $\phi, \psi$ are distributions of support $k$, and order $o$, then for $n=3, u(x, t) \subset C^{m}$ for $|x|<k_{2}$ and $t>T$ with $T=T\left(k_{1}, k_{2}, o, m\right)$.

We would really expect that the domain of smoothness would be defined by the interior of the envelope of all the characteristic cones issuing from within the singular support and then reflected from the body. However this has been shown so far only in special cases.

An alternative way of describing the smoothness theorem is that the solution operator acting on the Cauchy data and defined by solving the wave equation (1) and the boundary condition (5) is smoothing in the restricted sense that it takes rough initial data and smooths them in any finite region after sufficient time. An ultimate consequence of this is a theorem of Lax and Phillips, see [22], which gives the asymptotic description of the solution in exponential modes:

$$
u \sim \sum a_{i}(x) e^{\mu_{i} t} \text { as } t \rightarrow \infty, \quad x \in \mathcal{D},
$$

where $a_{i}(x)$ is a solution of $\Delta a_{i}-\mu_{i}^{2} a_{i}=0$ in $\varepsilon, a_{i}=0$ on $B$ with the special property that if $u_{i}$ satisfies (1) in free space and $u_{i}=a_{i}$, $u_{i t}=\mu_{i} a_{i}$ for $t=0$ then $\mu_{i}=0$ for $t<T(x)$.

The proof of the smoothness theorem leads us naturally into geometrical optics and so we sketch this proof before coming to Question III.

The solution $u(x, t)$ of (1), (3), (4), (5) can be represented in terms of the Riemann or source function $S(x, y, t)$ as

$$
u=\int\left(S \psi+S_{t} \phi\right) d y
$$

where $S$ satisfies

$$
\begin{array}{rlrl}
S_{t t}-\Delta_{x} S & =0, & & \text { in } \varepsilon, \\
S(0, x, y) & =0, & & \\
S_{t}(0, x, y) & =\delta(y-x), & \\
S(t, x, y) & =0, & \text { for } x \in ß .
\end{array}
$$


Thus if we know the behavior of $S$ for all values of $y$ it is easy to determine the behavior of $u$. The behavior of $S$ on the other hand can be found from its Fourier transform

$$
G=\int_{0}^{\infty} e^{i \lambda t} S d t
$$

which satisfies the reduced wave equation problem:

$$
\begin{aligned}
\Delta G+\lambda^{2} G & =\delta(x-y), \\
G=0, \quad \text { for } x \in \Theta, & \\
G_{r}-i \lambda G+G / r & \sim o\left(r^{-2}\right),
\end{aligned}
$$

with $r=|x|$.

The last condition is a variation of the Sommerfeld radiation condition which can be derived from it by using the far field expansion. At any rate if such a $G$ can be constructed and its transform taken it will yield the source function $S$. That there exists a Green's function for every real $\lambda$, follows from the general existence theorem established by Kupradse and Weyl. For bibliography and related existence theorems see Werner [14].

By inverting the transform one sees that the singular behavior of $S$ is determined by the behavior of $G$ for large $\lambda$.

On the other hand the high frequency behavior of the periodic solutions of the wave equation, Question III, also requires the asymptotic behavior of the Green's function $G$. Thus in either case we must settle the problem of geometrical optics.

4. Geometrical optics problem. We recollect that the general form of the geometrical optics solution, $G_{\mathrm{GO}}$ to (7) consists of the source $e^{i \lambda|x-y|}|| x-y \mid$ and a scattered wave. Thus

(9) $G_{\mathrm{GO}}=e^{i \lambda|x-y|} /|x-y|+e^{i \lambda \phi}\left(\chi_{0}+\lambda^{-1} \chi_{1}+\cdots+\lambda^{-N} \chi_{N}\right)$.

The function $\phi$ satisfies $|\nabla \phi|^{2}=1$ and is in fact distance along the reflected ray from the body. The functions, $\chi_{0}, \chi_{1}, \cdots$, each satisfy a first order ordinary differential equation along the reflected rays which is found by inserting $G_{\mathrm{GO}}$ in the differential equation of (8) and equating coefficients of the inverse powers of $\lambda$. They are uniquely determined by the reflecting boundary condition of (8) on $B$. In the shadow, see Figure 1, the second term reduces to $-e^{-i \lambda|x-y|} /|x-y|$ and $G_{\mathrm{GO}} \equiv 0$.

The differential equation is satisfied approximately since

$$
\left(\Delta+\lambda^{2}\right) G_{\mathrm{GO}}=\delta(x-y)+M \lambda^{-N+1} .
$$


The radiation condition of (7) is satisfied exactly because we chose the outward source for the first term.

This solution gives of course a particle picture of light emitted at a high frequency from a source at $y$ scattered in all directions and bounced from the body as if it were rigid and inelastic.

It appears that we could show that this particle or geometrical optics model is valid if $G-G_{\mathrm{GO}}=O\left(\lambda^{-N+1}\right)$. We would also for our present purposes be able to show that the source function $S \sim \int e^{-i \lambda t} G_{\mathrm{GO}} d \lambda$ and has the desired properties for the smoothness theorem.

However the quantity $M$ in (10) has highly singular properties where the shadow meets the illuminated region, see Figure 1. This affects the validity of geometrical optics and thus our proof.

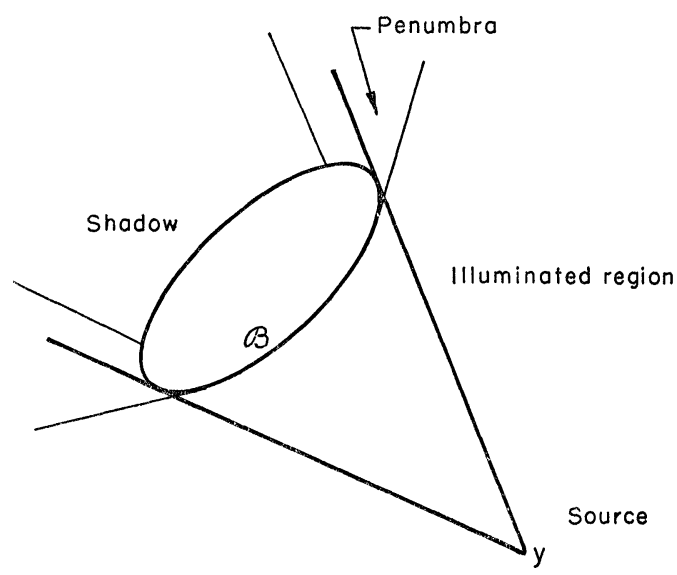

Figure 1

It is in fact necessary to construct a much smoother asymptotic solution which incorporates the diffraction behavior at the shadow edge. This was first accomplished for the three-dimensional case by Ludwig [15]. The two-dimensional problem was treated by Byslaev [16] through a boundary layer technique and by Grimshaw [17] following a method used by Ursell [18] in the water wave problem. Ludwig's method is applicable to any number of dimensions and has been extended to a wide class of equations and boundary values. However its technical details are lengthy so that we shall restrict ourselves to describing the properties we need. It is important to remember that it is not valid if the source is on the body. We denote this solution by $G_{L}$. It can be written in the penumbra as 


$$
G_{L}=e^{i \lambda|x-y|} /|x-y|+\int v(x, y, \lambda, \alpha) d \alpha
$$

where $v$ is a smooth asymptotic solution of the reduced wave equation. From the properties of $v$ one finds, see [11],

$$
\left(\Delta+\lambda^{2}\right) G_{L}=\delta(x-y)+M_{L} \lambda^{-N+2}, \quad \int r^{2} M_{L}^{2}|d x|<\infty,
$$

$$
\begin{gathered}
G_{L}=M_{L} \lambda^{-N / 3+7 / 6} \quad \text { on } \Theta, \\
\nabla G_{L} \cdot t=M_{L} \lambda^{-N / 3+13 / 6} \quad \text { on } \Theta, \quad \text { with } t \text { any tangent vector, } \\
G_{L} \partial / \partial r-i \lambda G_{L}+G_{L} / r=o\left(r^{-2}\right) .
\end{gathered}
$$

Here $M_{L}$ is bounded independent of $\lambda$ and $x$ for $x \in \varepsilon$.

Furthermore in the shadow and in the illuminated region but not in an arbitrary neighborhood (the penumbra) of the shadow edge $G_{\mathrm{GO}}-G_{L} \sim O\left(\lambda^{-N+1}\right)$.

Thus to establish geometrical optics in the lit and shadow regions we need only show that $G \sim G_{L}$. The proof is supplied by an appropriate estimating theorem, see [11]:

Estimating Theorem. Suppose $U$ has continuous derivatives in $\mathcal{E}$,

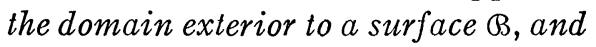

$$
\begin{gathered}
\Delta U+\lambda^{2} U=f \quad \text { in } \mathcal{E}, \\
U=g \quad \text { on } \beta, \\
\lim _{R \rightarrow \infty} \int_{r=R} r\left|U_{r}-i \lambda U+U / r\right|^{2} d \sigma=0 .
\end{gathered}
$$

Then if $x \cdot n \geqq \beta>0$ on $\beta$,

$$
|U(x)| \leqq A\left(\|f\| r^{-1 / 2}+\|\|\|\| r^{-1}\right)
$$

where $\|f\|^{2}=\int_{\varepsilon} r^{2}|f|^{2} d x \mid$ and $\||| g||^{2}=\lambda^{2}(\max |g|)^{2}+\int_{\Theta}|\nabla g|^{2} d \sigma$. The constant $A$ is independent of $\lambda$.

Substitution of $G_{L}-G$ for $U$ then yields the desired result using (11) since in our application the body is convex and hence strictly star-shaped. With a little refinement to improve the powers of $\lambda$ we finally obtain the following:

Asymptotic Theorem. $\left|G-G_{L}\right| \leqq M_{L} \lambda^{-N+3}$ with $M_{L}$ independent of $x$ and $\lambda$ and $\left|G-G_{\mathrm{GO}}\right|<M \lambda^{-N+3}$ where $M$ is bounded uniformly in every closed subdomain of $\varepsilon$ that excludes the shadow edge. 
It should be added that Ludwig's solution describes asymptotically the shadow field as it was originally described by Keller [19]. However the estimating theorem gives us no direct information about its asymptotic validity since both solutions are exponentially small in that region. However Bloom [20] has recently applied these estimates to prove the validity of the diffracted pattern in certain cases.

5. Proof of the estimating theorem. The estimating theorem is derived from energy estimates by using the fundamental solution $F=e^{i \lambda|x-y|} /|x-y|$ to obtain

$$
4 \pi U(x)=\int_{\varepsilon} F f|d y|+\int_{\mathbb{Q}}\left(\frac{\partial F}{\partial n} g-F \frac{\partial U}{\partial n}\right) d \sigma .
$$

Clearly if we could estimate $\partial U / \partial n$ on $\Theta$ say by $\int_{B}|\partial U / \partial n|^{2} d \sigma$ we could obtain the pointwise estimates for $U$ that are in the theorem. Equivalently since $U=g$ on $B$ we could use estimates of $\int_{\otimes}|\partial V / \partial n|^{2} d \sigma$ where $V=e^{-i \lambda r} U$. But $V$ satisfies by (12)

$$
-\Delta V=-(2 i \lambda / r)(r V)_{r}-e^{-i \lambda r f}
$$

and hence

or

$$
-2 \operatorname{Re}(r \bar{V})_{r} \Delta V=-2 \operatorname{Re}(r \bar{V})_{r} e^{-i \lambda r} f
$$

$$
-2 \operatorname{Re} \operatorname{div}(x \cdot \nabla \bar{V}) \nabla V+|\nabla V|^{2}=-2 \operatorname{Re}(r \bar{V})_{r} e^{-i \lambda r} f .
$$
have

Integrating over $\varepsilon$ and using the radiation condition from (12) we

$2 \operatorname{Re} \int_{\beta}(x \cdot \nabla \bar{V}) \frac{\partial V}{\partial n} d \sigma+\int_{\varepsilon}|\nabla V|^{2}|d x|$

$$
\leqq \frac{1}{100} \int_{\varepsilon} r^{-2}(r V)_{r}^{2}|d x|+100 \int r^{2} f^{2}|d x| .
$$

Applying the boundary condition and integrating the term on the right we find

$2 \operatorname{Re} \int_{\Theta}(x \cdot n)\left|\frac{\partial V}{\partial n}\right|^{2} d \sigma+\int_{\varepsilon}|\nabla V|^{2}|d x|+2 \operatorname{Re} \int_{\Theta}(x \cdot t)\left(e^{-i \lambda r} g\right)_{t} \frac{V \partial}{\partial n} d \sigma$

$$
\leqq \frac{1}{100} \int\left|V_{r}\right|^{2}|d x|+e,
$$

where $e \leqq\|f\|^{2}+\||g|\|^{2} ;()_{t}$ means tangential gradient and $t$ is a unit tangential vector. 
Applying Schwarz' inequality we find since $x \cdot n>\beta$,

$$
\beta \operatorname{Re} \int\left|\frac{\partial V}{\partial n}\right|^{2} d \sigma+\int|\nabla V|^{2}|d x| \leqq e .
$$

This proves the basic estimate leading to the estimating theorem.

To convert the behavior of $G$ for large $\lambda$ into the singular behavior of $S$ we have to study $G_{L}$ a little more closely in the penumbra but there is no real difficulty. To avoid moving the source right up to the body requires some tricks which are described in [12] and in [13].

This completes the main results for the first three questions. The scattering question is intimately related to both decay and smoothness. The solution at infinity does behave like a free space solution, see Friedlander [21], and thus one can consider what one really has in the operator $\mathfrak{M}^{\prime} \mathfrak{N}^{-1}$. This subject has been treated at length in a monograph by Lax and Phillips [22] and the reader is referred to the relevant chapters.

Appendix 1. Rates of decay. A rate of decay for the energy is most easily found by finding a suitable conservation identity. Let us look at the one-dimensional problem $u_{t t}-u_{r r}=0$. Multiplying by $2 u_{t}$ yields the identity

$$
\left(u_{t}^{2}+u_{r}^{2}\right)_{t}-\left(2 u_{r} u_{t}\right)_{r}=0
$$

or on integrating

$$
\oint\left(u_{t}^{2}+u_{r}^{2}\right) d r+2\left(u_{r} u_{t} d t\right)=0
$$

for any closed curve. From this identity one gets energy conservation. But under any change of coordinates a vanishing line integral remains a vanishing line integral and hence if the transformation leaves $u$ a solution of the wave equation in the transformed coordinates we may possibly obtain a new vanishing line integral (or conservation identity) for $u$. The obvious shift transformation $t \rightarrow a t^{\prime}+b, r \rightarrow a r^{\prime}+b$ yields the energy identity again. However the Kelvin transformation $r^{\prime}=r /\left(r^{2}-t^{2}\right), t^{\prime}=t /\left(r^{2}-t^{2}\right)$ yields the new conservation identity (on dropping the primes)

$$
\oint\left\{\left[(r+t)^{2}\left(u_{r}+u_{t}\right)^{2}+(r-t)^{2}\left(u_{r}-u_{t}\right)^{2}\right] d r+4 r t\left(u_{t}^{2}+u_{r}^{2}\right) d t\right\}=0 .
$$

This identity could also be obtained by considering the transformation ${ }^{4}$ of the multiplier $u_{t}$ which is $\left[\left(r^{2}+t^{2}\right) u_{t}+2 r t u_{r}\right] /\left(r^{2}-t^{2}\right)^{2}$ and applying it directly to the transformed domain.

See Morawetz [23] and [22]. 
One can generate another identity by applying a shift to our new identity. This, incidentally, exhausts the set of vanishing line integrals whose integrands are quadratic in the derivatives.

Clearly the same principles ${ }^{5}$ apply in higher dimensions and one gets in fact identities involving the angular derivatives as well as derivatives with respect to $r$, the distance outward and in. Thus in three dimensions the multiplier is $\left(r^{2}+t^{2}\right) u_{t}+2 r t u_{r}+2 t u$. We present here only the integrated form for three dimensions where the integration has been carried out in $\varepsilon$ for $0<t \leqq T$ on a solution $u$ of (1), (3), (4) and (5). Thus if the solution $u$ vanishes on $B$,

$$
\begin{aligned}
\int_{t=T}\left[(r+t)^{2}\left(u_{r}+u_{t}\right)^{2}+(r-t)^{2}\left(u_{r}-u_{t}\right)^{2}\right. & \\
& \left.+2\left(r^{2}+t^{2}\right)\left(|\nabla u|^{2}-u_{r}^{2}\right)\right]|d x| \\
& +\int_{0}^{T} \int_{B}(x \cdot n)\left(\frac{\partial u}{\partial n}\right)^{2} d \sigma d t=\text { constant. }
\end{aligned}
$$

Here $n$ is a unit normal into $\varepsilon$ from $\leftrightarrow$. The constant can be evaluated and is bounded by $k^{2} E(0, \varepsilon)$.

Since the body is star-shaped, $x \cdot n \geqq 0$ so that both integrands are positive and thus both integrals are bounded by $k^{2} E(0, \varepsilon)$. Hence the contribution to the first integral from $|x|<K$ is also bounded. But there $(r+t)^{2}>t^{2} / 2,(r-t)^{2}>t^{2} / 2,\left(r^{2}+t^{2}\right)>t^{2} / 2$ for $t$ large enough. Hence

$$
\int_{t=T ;|x|<K} t^{2}\left(|\nabla u|^{2}+u_{t}^{2}\right)|d x|
$$

being bounded by this contribution is also bounded by $k^{2} E(0, \varepsilon)$. Thus we have $E(t, D)<(k / t)^{2} E(0, \varepsilon)$ if for $D,|x|<K$.

This completes the proof of energy decay for any dimension.

Appendix 2. Exponential decay. On the other hand exponential decay is rooted in Huyghens' principle and cannot be proved so simply. One method of proving it involves the following lemma which describes the breaking up of the solution and depends on the principle.

Lemma. Suppose u satisfies the Cauchy boundary value problem for $' \geqq 0$. The support of $B$ is $k$ and of the data $3 k$. Then, for $t>T>3 k$, $u=u_{B}+u_{F}$ where

(a) $u_{F}$ is a free space solution that vanishes for $|x|<t-T-k$,

${ }^{5}$ See E. Noether [24], for a related invariance principle. 
(b) $u_{B}$ satisfies the original Cauchy boundary value problem for $t \geqq T$ $+2 k$ with data of support $3 k$ for $t=T+2 k$.

To get exponential decay we now suppose that there exists a rate of decay, i.e., for any solution of initial support $3 k, E(t, D)<f(t) E(t, \mathcal{E})$ for $D$ the sphere $|x|<3 k$, and $\lim _{t \rightarrow \infty} f(t)=0$. Let $E(t, \varepsilon)=1$. Then for $u_{B}$ at $t=T, E(t, \varepsilon)<f(t)$.

Using some domain of dependence arguments and Schwarz' inequality one finds in fact a constant $a$ such that for $u_{B}$ at $t=T+2 k$, $E(t, \varepsilon)<a f(t)$.

Next, start with $u_{B}$ at $t=T+2 k$ and move forward another time step. Perform the same break up into let us say $u_{2 B}, u_{2 F}$ with $u_{B}$ $=u_{2 B}+u_{2 F}$. Then by the rate of decay at time $2 T+4 k$ the solution $u_{2 B}$ will have $E(t, \varepsilon)<a^{2} f^{2}(t)$.

Continuing, we eventually have near the body $u=u_{N B}$ where $u_{N B}$ has energy less than $(a f(T))^{N}=\exp N \log a f(T)$ for $t>N(T+2 k)$. By choosing $T$ large enough log $a f(T)<0$ and we have the energy of $u$ in $|x|<3 k$ for $t>N(T+2 k)+2 k$ equal to the energy in $u_{N B}$ where $N$ is the largest integer such that $N(T+2 k) \leqq t$. Thus $N \sim t / T+2 k$ and hence the energy is decaying exponentially.

We prove the lemma for $u=0$ on $B$ and $u \subset C^{\infty}$. We choose $u_{B}$ to satisfy (1) in $\varepsilon$ for $t \geqq T$ and $u_{B}=u, u_{B t}=u_{t}$ for $|x| \leqq k, t=T$ and $u_{B}=u_{B t}=0$ for $|x| \geqq k, t=T$. The data for $u_{B}$ should really be smoothed at $|x|=k$ but we shall ignore this fractional correction.

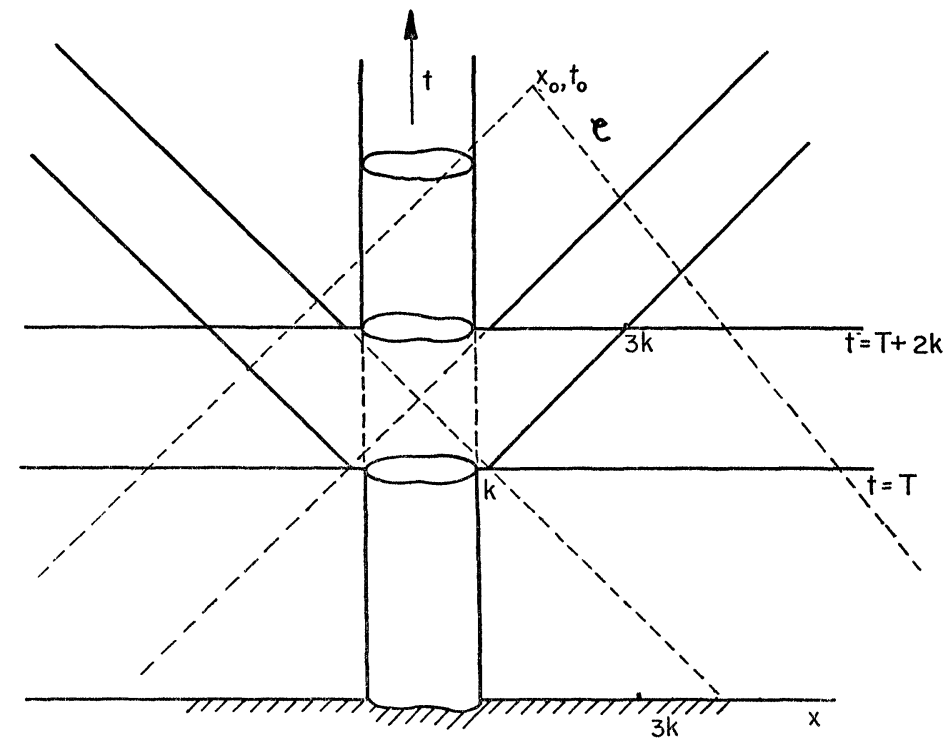

FIGURE 2 
Then $u_{F}=u-u_{B}$ is chosen to be a free space solution for $t \geqq T$ with zero data for $|x|<k$. For $t<T, u_{F}$ is set equal to $u$. Looking at Figure 2 , one sees that the whole characteristic cone dropped backward from $x_{0}$ lies in a region where $u_{F}$ is a smooth solution of $\square u=0$. Hence we can apply the Riemann function to find that $u_{F}\left(x_{0}, t_{0}\right)$ depends only on the initial data of $u$ outside the support $3 k$. Hence $u_{F}\left(x_{0}, t_{0}\right)=0$ which gives (a). Part (b) follows by noting that in the region where $u_{F}=0$ on $B$ we will have $u_{B}=u-u_{F}=u=0$.

\section{REFERENCES}

1. R. Courant, Methods of mathematical physics. Vol. II: Partial differential equations, Interscience, New York, 1962. MR 25 \#4216.

2. Walter A. Strauss, Decay and asymptotics for $\square \mathrm{u}=F(u)$, J. Functional Analysis 2 (1968), 409-457. MR $38 \# 1385$.

3. P. D. Lax and R. S. Phillips, The wave equation in exterior domains, Bull. Amer. Math. Soc. 68 (1962), 47-49. MR 24 \#A913.

4. N. A. Schenk, Eigenfunction expansions and scattering theory for the wave equation in an exterior region, Arch. Rational Mech. Anal. 21 (1966), 120-150.

5. D. M. Ėdus, The principle of limiting absorption, Mat. Sb. 57 (99) (1962), 13-44; English transl., Amer. Math. Soc. Transl. (2) 47 (1965), 157-191. MR 26 $\# 2722$.

6. J. Ralston, Solutions of the wave equation with localized energy, Comm. Pure Appl. Math. (1969) (to appear).

7. C. Wilcox, The initial-boundary value problem for the wave equation in an exterior domain with spherical boundary, Notices Amer. Math. Soc. 6 (1959), 869-870. Abstract \#564-240.

8. P. D. Lax, C. S. Morawetz and R. S. Phillips, Exponential decay of solutions of the wave equation in the exterior of a star-shaped obstacle, Comm. Pure Appl. Math. 16 (1963), 477-486. MR 27 \#5033.

9. C. S. Morawetz, The limiting amplitude principle, Comm. Pure Appl. Math. 15 (1962), 349-361. MR 27 \#1696.

10. J. B. Keller, R. M. Lewis and B. D. Seckler, Asymptotic solution of some diffraction problems, Comm. Pure Appl. Math. 9 (1956), 207-265. MR 18,43.

11. C. S. Morawetz and D. Ludwig, An inequality for the reduced wave operator and the justification of geometrical optics, Comm. Pure Appl. Math. 21 (1968), 187-203. MR $36 \# 6185$.

12. - The generalized Huyghens' principle for reflecting bodies, Comm. Pure Appl. Math. 22 (1969), 189-205.

13. R. S. Phillips, A remark on the preceding paper of C. S. Morawetz and D. Ludwig, Comm. Pure Appl. Math. 22 (1969), 207-211.

14. P. Werner, Randwertprobleme der mathematischen Akustik, Arch. Rational Mech. Anal. 10 (1962), 29-66. MR 26 \#5276.

15. D. Ludwig, Uniform asymptotic expansion of the field scattered by a convex object at high frequencies, Comm. Pure Appl. Math. 20 (1967), 103-138. MR 34 \#3879.

16. V. S. Buslaev, Short-wave asymptotic behaviour in the problem of diffraction by smooth convex contours, Trudy Mat. Inst. Steklov. 73 (1964), 14-117. (Russian). MR 31 \#4346.

17. R. Grimshaw, High-frequency scattering by finite convex regions, Comm. Pure Appl. Math. 19 (1966), 167-198. MR 33 \#2107. 
18. F. Ursell, On the short-wave asymptotic theory of the wave equation $\left(\nabla^{2}+k^{2}\right) \phi$ =0, Proc. Cambridge Philos. Soc. 53 (1957), 115-133. MR 18,848.

19. J. B. Keller, Geometrical theory of diffraction, J. Opt. Soc. Amer. 52 (1962), 116-130. MR 24 \#B1115.

20. C. O. Bloom, On the validity of the geometrical theory of diffraction by star shaped cylinders, Notices Amer. Math. Soc. 16 (1969), 512. Abstract \#664-39.

21. F. G. Friedlander, On the radiation field of pulse solutions of the wave equation. II, Proc. Roy. Soc. Ser. A 279 (1964), 386-394. MR 29 \#1431.

22. P. D. Lax and R. S. Phillips, Scattering theory, Pure and Appl. Math., vol. 26, Academic Press, New York, 1967, Chap. 5, pp. 151-165. MR 36 \#530.

23. C. S. Morawetz, Energy identities for the wave equation, New York University. Inst. Math. Mech. 346, January 1966.

24. - Exponential decay of solutions of the wave equation, Comm. Pure Appl. Math. 19 (1966), 439-444. MR 34 \#4664.

25. E. Noether, Invariante Variationsprobleme, Nachr. Ges. Göttingen Math.Phys. Kl. 1918, 235-257.

Courant Institute of Mathematical Sciences, New York University, NEW York, New York 10012 